\title{
INSTITUTO SINGULARIDADES
}

NÚCLEO DE ALTO RENDIMENTO ESPORTIVO DE SÃO PAULO - NAR-SP

HOMAR FAYÇAL CAMPOS COSTA

USO DA TERMOGRAFIA NAS ANÁLISES DOS DANOS MUSCULARES EM ATLETAS DE KARATE

SÃO PAULO

2019 
INSTITUTO SINGULARIDADES

NÚCLEO DE ALTO RENDIMENTO ESPORTIVO DE SÃO PAULO - NAR-SP

HOMAR FAYÇAL CAMPOS COSTA

\title{
USO DA TERMOGRAFIA NAS ANÁLISES DOS DANOS MUSCULARES EM ATLETAS DE KARATE
}

\author{
Trabalho apresentado ao Instituto \\ Singularidades como requisito parcial para \\ conclusão do curso de Especialização em \\ Metodologia do Treinamento para o Esporte de \\ Alto Rendimento.
}

Orientador: Prof. Dr. Cesar Cavinato Cal Abad

SÃo PAULO

2019 


\section{SUMÁRIO}

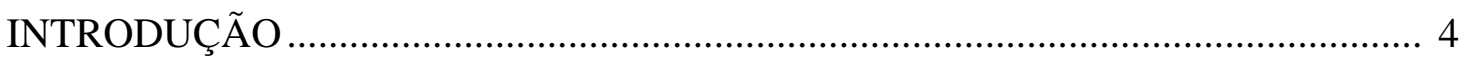

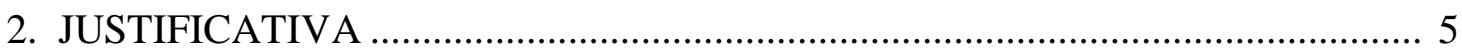

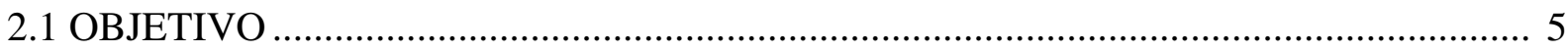

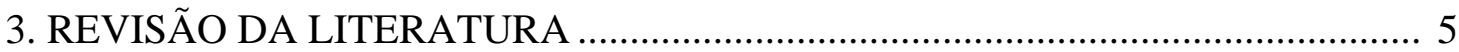

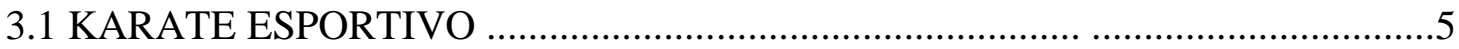

3.2 TRAUMAS MUSCULARES NO KARATE...................................................6

3.3 TERMOGRAFIA NA ANALISES DOS TRAUMAS MUSCULARES...........................................

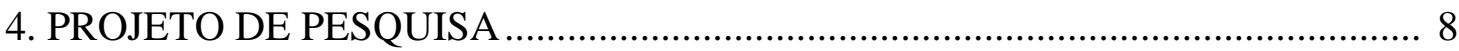

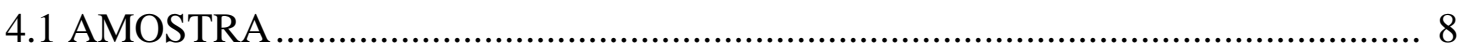

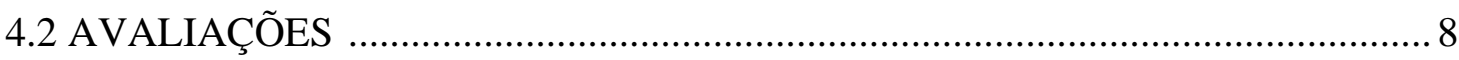

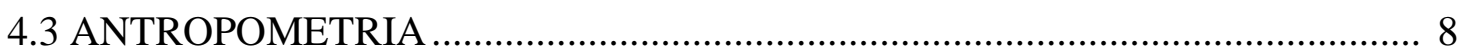

4.4 MONITORAMENTO DA FREQUÊNCIA CARDÍACA (FC) ................................

4.5IMAGEM TERMOGRÁFICA (IT) ...................................................... 9

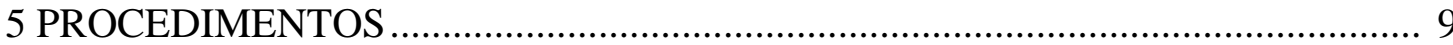

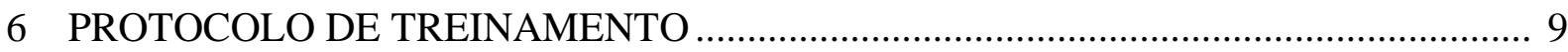

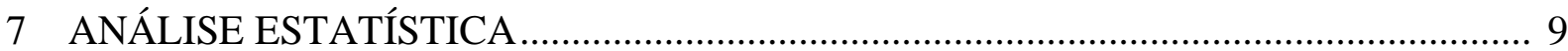

8 RESULTADOS ESPERADOS ................................................................................... 10

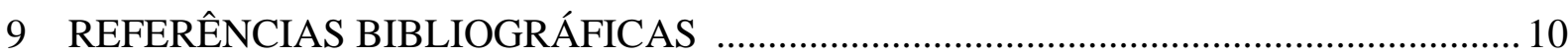




\section{1 - INTRODUÇÃO}

A temperatura corporal é um bom indicador da saúde humana. O sistema de imagem térmica (termografia) fornece orientação visual (documentação) e qualitativa de mudanças de temperatura nos tecidos vasculares, e está começando a desempenhar um papel importante no campo esportivo (TAN, et al 2009). A termografia é um método não invasivo utilizado para registrar padrões térmicos corporais, usando a câmera termográfica $(\mathrm{CT})$, sendo utilizada para medir a radiação térmica (calor) emitida pelo corpo ou partes deste, podendo, portanto, ser utilizada para diagnóstico de danos musculares causadas pelo treinamento e jogos oficiais (BANDEIRA, et al 2012; KITCHEN, S. et al, 1998).

A termografia é reconhecida com método diagnóstico pela American Medical Association desde 1987 (NEVES, 2014). Este método identifica por meio de radiação infravermelha emitida pela pele, e proporcionando assim a análise das funções fisiológicas relacionadas com o controle da temperatura da pele de forma não invasiva, sem expor o individuo a qualquer tipo de radiação. Vale destacar que o treinamento de alto desempenho leva o sistema locomotor para seus limites fisiológicos (HILDEBRANDT, 2010) e a termografia pode ser uma ferramenta de acompanhamento desse processo.

Logo após a um treino de alta intensidade ou jogo oficial, à alta sensibilidade a alterações fisiológicas (temperatura) musculares, o uso de imagens térmicas em fusão com imagens de ressonância magnética tem sido recomendado para o diagnóstico e acompanhamento de dano ou lesões musculares (SANCHES, 2013). A temperatura da pele registrada pela termografia pode ser influenciada por diferentes fatores, como massa muscular e camada de gordura subcutânea. Podendo ser indicado a ser feito em indivíduos com camada de gordura subcutânea inferior ou com baixa porcentagem de gordura corporal, tendo uma taxa de variação mais alta na temperatura da pele durante o exercício do que aqueles com camada de gordura subcutânea superior 20\% de massa gorda, (FELISBERTO, 2015).

A inflamação muscular resultante da força reflete o dano muscular (destruição das fibras musculares e da libertação de metabolitos) que ocorre durante a contração muscular realizado com cargas elevadas (NEVES, 2015). Este processo também envolve variações no volume de fibras musculares, a libertação de marcadores bioquímicos e de produção de calor local (SANCHES, 2013). Existem diversos métodos relatados na literatura para análise e diagnóstico de traumas musculares causadas pela atividade física. Entre esses métodos, destacam-se como os mais utilizados: atividade de enzimas plasmáticas, lactato desidrogenase (LDH), troponina I e mioglobina, aquisição das respostas subjetivas de dor por meio de escala de percepção (BANDEIRA, 2014).

A temperatura corporal é controlada pelo hipotálamo. Os três principais fatores que determinam sua variação são: taxa metabólica basal, atividade orgânica específica e atividade muscular. Os mecanismos físicos da condução térmica são: condução, convecção, evaporação e radiação. A convecção, por meio do fluxo sanguíneo, é o principal mecanismo de transferência de calor dentro do corpo. Por outro lado, a radiação (infravermelha na faixa espectral de 0,7 a $15 \mu \mathrm{m}$ ) é o maior mecanismo de perda térmica do corpo humano, responsável por $60 \%$ da perda total de calor (BRIOSCHI, 2005).

Com relação aos tecidos corporais, podemos entender, de forma simplificada, que os músculos e orgãos internos são os locais de geração de calor; o sangue é o principal tecido responsável pela dissipação do calor e o tecido adiposo desempenha uma função de isolante térmico (BRIOSCHI, 2005 e BANDEIRA, 2014) 


\section{2 - JUSTIFICATIVA}

Considerando que o treinamento de alto desempenho leva o sistema locomotor para a borda de seus limites fisiológicos e com sucessivos danos musculares que podem culminar com lesões mais graves, a termografia pode ser uma ferramenta de acompanhamento desse processo para prevenir lesões, especialmente musculares (NEVES et al, 2014). Sua utilização como diagnóstico de lesões musculares após treinamento justifica-se por ser uma técnica não invasiva e de fácil aplicação.

A termografia é um método que auxilia no diagnóstico de lesões musculares que tem se mostrado eficiente na avaliação de lesões causadas pelo esporte. A alteração de temperatura apresentada pela pele em função da inflamação muscular possibilita a mensuração e avaliação do nível inflamatório por meio do gradiente de temperatura possibilitando a localização da área lesada. Um exemplo é o estudo realizado por Brancaccio (2006), onde houve a comparação de dois métodos de avaliação de lesão muscular: um método avaliando a presença de creatinaquinase no sangue $24 \mathrm{~h}$ após seções de treino ou pós-jogo nos atletas, e outro com marcadores do processo inflamatório medidos também 72hs (BRANCACCIO, 2007). Em outro estudo, De Souza (2010), encontrou valores de CK em repouso em atletas adultos de futebol de salão de $256,1 \pm 23,6 \mathrm{U} / \mathrm{L}$, enquanto que após o término da primeira e segunda partida, os valores encontrados foram de 372,6 $\pm 53,4$ e 408,8 $\pm 68,8 \mathrm{U} / \mathrm{L}$, respectivamente. O grupo experimental apresentou uma diferença estatisticamente para os três músculos estudados, sugerindo a possibilidade da utilização de imagem termográfica em conjunto com a creatina-quinase. Por esse método, os autores recomendaram a utilização da termografia para determinação da localização de lesões musculares pós-treino, pois só a creatina-quinase, mesmo estando em altas concentrações no sangue, não é sensível para determinar a região anatômica onde poderá ocorrer lesão muscular.

O uso da termografia para diagnostico de lesões no esporte é relatado em estudos com populações de jogadores de futebol e nadadores (BANDEIRA 2012). Entretanto, há uma escassez de estudos na população de atletas de alto rendimento de esportes individuais, como é o exemplo dos atletas de Artes Marciais. Tais atletas tem alta propensão a danos musculares no período de treinamento por se tratar de esporte de contato e de alta intensidade.

\section{1 - OBJETIVO}

O objetivo deste projeto de pesquisa é verificar a possibilidade da utilização da termografia nos traumas musculares causados pelo treinamento físico e nas competições de karate.

\section{REVISÃO DA LITERATURA}

\section{1 - KARATE ESPORTIVO}

O Karatê é uma arte marcial japonesa com golpes de percussão de média distância, onde em treinamento executa-se o Kumite (luta), Kihon (forma) e o Kata (luta imaginária), Paralelamente com essa prática, o Karateca também possibilita trabalhar as qualidades físicas de base (força, resistência, velocidade e flexibilidade) (SANTINA, 2016). O Karatê é um exemplo de arte marcial de alto rendimento e é uma das mais populares artes marciais praticadas no mundo (MILANEZ, 2012). A modalidade envolve movimentos complexos realizados por diversos grupos musculares e com grandes variações de acelerações e desacelerações. As técnicas de curta duração de ataque e defesa caracterizam-se por execuções com máxima intensidade, interrompidas por pequenos intervalos. Isso faz com que a modalidade tenha característica intermitente. (MILANEZ, 2012).

Atualmente, o nível competitivo do karate aumentou exponencialmente, o que tem gerado um maior profissionalismo da modalidade. Além disso, a importância do esporte é reconhecido pelo Comitê Olímpico Internacional que reconhece o karatê como uma modalidade 
olímpica com a sua primeira apresentação nos próximos Jogos Olímpicos de Tóquio 2020, (Spigolon, 2018). Segundo a Federação Mundial de Karatê (WKF, 2018), o Karate é dividido em duas categorias de disputa que consistem em kata (demonstração) e kumite (combate). Ambas as categorias são classificadas em individuais, por sexo, idade e peso.

De Oliveira (2011) em seu estudo, determinou que os tipos mais frequentes de lesões em atletas de alto nível no karatê são as lesões nos membros inferiores $(55,9 \%)$ seguida pelos membros superiores $(28,4 \%)$. Quanto maior o tempo de prática na modalidade, mais suscetível à danos musculares o atleta pode ter, porém esse não parece ser o único motivo da aparição de uma injúria, quanto maior a experiência de um atleta na modalidade, mais confiança na realização da técnica ele irá ter, fator que demonstra aumentar as chances de uma lesão, principalmente no decorrer do combate, no karatê, o chutes laterais (mawashi e Ura Mawashi) são os golpes mais utilizados, logos os membros inferiores são os locais mais acometidos por danos musculares (Santos, 2015)

\section{2 - TRAUMAS MUSCULARES NO KARATE}

(DE OLIVEIRA, 2011) relata em seu estudo que atletas de Karate ao preencherem um questionário contêm algumas informações relevantes dos atletas, observou que o percentual de lesões no grupo estudado foi de $77,5 \%$, sem diferença estatística entre os gêneros $(77,6 \%$ homens e 72,3\% mulheres; $\mathrm{p}=0,9701, \mathrm{X} 2$ ). As lesões foram mais frequentes nos membros inferiores $(55,9 \%)$ seguida pelos membros superiores $(28,4 \%)$.

O Karate também possui a sua parte esportiva, onde em uma competição de combate, vence o atleta que golpear o adversário primeiro com movimentos técnicos da modalidade, podendo ser socos e chutes, na tentativa de golpear o corpo do oponente e, assim, causar um impacto, garantido a pontuação.

A situação de combate no Karatê-do aumenta muito as chances de uma LD (lesões Desportivas) acontecer, sendo objeto de pesquisa de alguns estudos que buscam entender melhor esse fenômeno, como a pesquisa realizada por Zetaruket al.(2005), que relata que já é possível detectar esta tendência à lesão, entrevistando 263 praticantes de diversos estilos de artes marciais diferentes, com a sua maioria adepta ao Karate, e identificando que o Karate é a segunda arte marcial com mais LD, tendo a sua prevalência nos membros inferiores, sendo observado também que atletas com maior tempo de experiência sofrem ou já sofreram algum tipo de lesão e destacando lesões de membros inferiores.

JUNIOR (2012) relata que o membro inferior foi o local com maior lesão, tendo um percentual de $36 \%$, em segundo ficou o membro superior com $24 \%$, depois a face com $15 \%$, em quarto o tronco com $13 \%$, em quinto o pescoço com $8 \%$, em sexto a cabeça com $4 \%$ e em último com $0 \%$ a mão, o pé, o tornozelo e o punho.

SOUZA (2011) relata e faz um comparativo entre atletas de karate e Jiu-Jitsu e verificou que as pernas foi o terceiro local com mais frequência de lesões no Karate Shotokan e apresentou uma incidência significativamente maior $(\mathrm{p}=0,042)$ quando comparado aos praticantes de Jiu-Jitsu; ou seja, do total de lesões na perna, 77,8\% ocorreram no Karate Shotokan contra $22,2 \%$ no Jiu-Jitsu. Já ao compararmos os locais de lesão nos praticantes de Jiu-Jitsu, observou-se uma frequência significativamente maior no joelho, ombro e orelha apresentando $\mathrm{p}=0,000$ para os três locais. Do total de lesões de joelho, $72,2 \%$ ocorreram no Jiu-Jitsu contra 27,8\% no Karate Shotokan; já no ombro, a prevalência foi de $76,7 \%$ no JiuJitsu e de 23,3\% no Karate Shotokan; e, na orelha, a diferença foi alarmante, sendo 95,2\% no Jiu-Jitsu contra 4,8\% no Karate Shotokan.

CRITCHLEY, (1999) documenta em seu artigo que a taxa de lesões em três campeonatos britânicos de karatê Shotokan nos anos de 1996, 1997 e 1998, nesses torneios, com as regras restritas onde o contato, com permissão somente "leve" ou "toque", teve o registro de lesões após 1770 lutas nas três competições nacionais, num total de 160 lesões ocasionadas 
durante o ataque do competidor, onde $57 \%$ das lesões foram na cabeça. A idade média dos feridos foi de 22 anos, com uma média de nove anos de experiência no karate.

ZIAEE (2015) estudou 620 atletas onde a taxa de incidência de lesões por atleta foi de $16,1 \%$ e 20,2 por 100 atletas. Noventa por cento dos ferimentos ocorreram durante a prática de ataque, $6 \%$ durante o treino de rotina e $4 \%$ durante o kata. A taxa de lesão foi mais comum em atletas com peso inferior a $70 \mathrm{~kg}$ e com menor experiência esportiva $(\mathrm{P} \leq 0,05)$. Os locais mais comuns para lesão foram cabeça e pescoço seguidos por tronco, membro inferior e superior, respectivamente. Apenas 2 casos necessitaram de intervenção cirúrgica e ninguém levou a diminuição do nível de consciência. O tipo mais comum de lesão foi contusões e raspões superficiais $(64 \%)$.

\section{3 - TERMOGRAFIA NA ANALISES DOS TRAUMAS MUSCULARES}

Na Medicina do Esporte, o uso da termografia pode proporcionar melhores resultados aos atletas por ser um instrumento na identificação de riscos e na prevenção de lesões, além de ser uma importante ferramenta no acompanhamento do treinamento esportivo, a partir da avaliação da quantificação da carga de trabalho (Neves, 2014). Os atletas são expostos a um estresse físico do treinamento e de competições. Reações por sobrecarga são frequentes; portanto, diagnósticos precoces são importantes. Além do diagnóstico precoce, a localização da inflamação é um passo fundamental no tratamento correto. A termografia também possibilita monitorar a temperatura da superfície corporal antes, durante e após o movimento e detectar mudanças na temperatura da pele causadas pelo exercício (FERREIRA, 2008).

A circulação periférica tem uma importante função no tratamento de lesões e na termorregulação. Para diferenciar as mudanças de temperaturas por lesões das mudanças de temperatura pela sobrecarga de exercício, é preciso compreender as diferentes respostas fisiológicas das estruturas envolvidas (CHAUDHRY, 2016).

Através de um ensaio clínico controlado, Bandeira, Moura, Souza, Nohama e Neves (2012) buscaram avaliar a concentração sérica de CK, lactato e a verificar imagem de infravermelho de quadríceps femoral de 18 atletas do sexo masculino, com idades entre 15 e 17 anos, que treinam regularmente na equipe juvenil de futebol do Paraná Clube.

Por sua vez, Carmona (2012) publicou um estudo demonstrando a aplicabilidade da termografia na prevenção de lesões no futebol. O protocolo apresentado indicava, de acordo com a análise dos termogramas dos jogadores, se o mesmo estava liberado para o treino, se deveria fazer um treino mais leve, ou se deveria ir para a fisioterapia. Esse protocolo conseguiu reduzir significativamente a incidência de lesões musculares na equipe considerada durante a temporada de 2009.

Em um recente, trabalho de pesquisa, Bandeira et al (2014), avaliaram o impacto dos treinos e jogos em diversos grupos musculares de atletas de rúgbi, concluindo que no grupo de atletas que apresentaram elevação da CK superior a 50\% entre o primeiro e o segundo momento de avaliação, os músculos peitorais do lado esquerdo e semitendinoso do lado esquerdo apresentaram diferenças de temperatura significativas ( $\mathrm{p}$ de 0,037 e 0,045 ), respectivamente. E ainda, que esses músculos são os mais afetados numa partida desse esporte, e que a termografia é um método válido para identificação de lesões musculares.

Um estudo interessante, foram avaliados jogadores de voleibol antes e após o treino, houve uma correlação estatisticamente significativa entre o consumo máximo de oxigênio (VO2máx.) e uma diminuição na temperatura da superfície dos membros superiores ( braço e antebraço ) imediatamente após o treino físico. Os autores sugeriram que a termografia pode ser usada como um método adicional, não invasivo, de estimar o estado de condicionamento aeróbio de um atleta em comparação a outros (CHUDECKA et al., 2012). 


\section{4 - PROJETO DE PESQUISA}

Nos artigos de (CHUDECKA et al., 2012; CARMONA, 2012; BANDEIRA et al, 2012; BANDEIRA et al, 2013; BANDEIRA et al, 2014; NEVES et al, 2014) destacam que o treinamento de alto desempenho leva o sistema locomotor para a borda de seus limites fisiológicos e a termografia pode ser uma ferramenta de acompanhamento desse processo. Sua utilização como diagnóstico de traumas musculares após treinamento justifica-se por ser uma técnica não invasiva e de fácil aplicação. Caso a hipótese esteja correta, será possível localizar com facilidade os pontos de inflamação muscular decorrentes do protocolo de luta que será aplicado neste projeto. Sendo assim, o objetivo deste estudo será verificar a possibilidade da utilização da termografia no diagnóstico de traumas causados pelo treinamento físico e competições de caratecas.

\section{1- $\quad$ AMOSTRA}

O estudo será realizado com 59 atletas do sexo masculino e feminino, pertencentes ao Projeto São Paulo Olímpico - FPK e Seleção Paulista de Karate. Com idade que se enquadrem na categoria "sênior" de 18 a 24 anos, sendo a idade média dos atletas masculinos de 21,3 anos e para o as atletas femininas, 18,03, anos, que treinam regularmente nas suas equipes e que estejam no circuito nacional e Internacional de competição da Federação Mundial de Karate (World Karate Federation - WKF).

\section{2- $\quad$ AVALIAÇÕES}

As avaliações e as imagens serão adquiridas antes e depois do protocolo de treino, exceto a antropometria, na ordem a seguir:

a) Antropometria (estatura, peso, diâmetros corporais, perímetros e dobras cutâneas) será realizada no dia anterior ao protocolo de treino para perfil dos atletas;

b) Monitoramento da frequência Cardíaca;

c) Imagem termográfica (pré e pós-intervenção)

Tabela 1: testes pré e pós-intervenção

\begin{tabular}{l|l}
\hline Sequência & Testes pré-intervenção \\
\hline 1 & Antropometria - um dia antes da intervenção \\
\hline 2 & Imagem termográfica pré-intervenção (antes luta) ou treino \\
\hline Sequência & Testes pós-intervenção \\
\hline 1 & Imagem termográfica pós-intervenção (antes luta) ou treino \\
\hline
\end{tabular}

\section{3- ANTROPOMETRIA}

A avaliação antropométrica será realizada um dia antes dos protocolos de intervenção (GC e GT) com intuito de traçar o perfil dos atletas. As medidas serão coletadas como segue abaixo, por um avaliador experiente.

a) Peso corporal (Balança);

b) Estatura corporal (estadiômetro);

c) Circunferência corporal: abdômen, quadril, coxa, braço e perna (fita métrica com precisão de $0,1 \mathrm{~cm}$ );

d) Dobras cutâneas: subescapular, tríceps, axilar média, supra ilíaca, abdominal, coxa e perna (compasso de dobras cutâneas); 


\section{4 - MONITORAMENTO DA FREQUÊNCIA CARDÍACA (FC)}

A FC será monitorada durante todo o protocolo de intervenção para monitoramento da intensidade de esforço (monitor de frequência cardíaca).

\section{5 - IMAGEM TERMOGRÁFICA (IT)}

$\mathrm{Na}$ avaliação pré-treino, os sujeitos do GC e CT deverão permanecer de 15 a 30 minutos no ambiente controlado com a área do corpo a ser avaliada descoberta, para fins de aclimatação, pois se sabe que a temperatura da pele varia com a temperatura do ambiente (NEVES et al., 2014). Após o período de aclimatação, será realizada uma captura de IT do quadríceps femoral, peitoral e ombro.

Na avaliação pós-treino, os sujeitos do GC e GT serão avaliados aproximadamente 24 horas após o treinamento. Coleta das medidas da dobra cutânea da coxa e peitoral dos atletas para fins de verificação da influência desta variável na temperatura medida pela câmera.

\section{PROCEDIMENTOS}

Os atletas serão divididos em dois grupos, sendo um denominado de controle (GC) e outro denominado grupo experimental (GT). Ambos os grupos treinarão da seguinte forma: $\mathrm{O}$ GC participará de um treinamento de baixa intensidade enquanto o GT participará de uma sessão de treinamento de alta intensidade. Ambos os grupos farão imagens termográficas.

\section{PROTOCOLO DE TREINAMENTO}

Os atletas do GT realizarão 05 lutas de 3 minutos cronometrados dentro do treinamento convencional, simulando o ambiente mais próximo possível de uma competição com os devidos intervalos entre cada luta 5 minutos de intervalos. O exercício consiste em realizar as lutas com um adversário da mesma categoria de peso. Para que a intensidade do exercício chegue ao máximo de seu desempenho simulando a competição em quase sua totalidade os atletas serão encorajados verbalmente pelo pessoal de apoio.

\section{ANALISE ESTATÍSTICA}

As imagens serão adquiridas utilizando-se uma câmera produzida pela FLIR Inc.®, modelo A-325. Esta câmera tem resolução integrada de 320x240 pixels e sensibilidade para detectar diferenças de temperatura menores de $0,08^{\circ} \mathrm{C}$. A câmera é ligada por meio de um cabo de rede a um computador com software específico instalado para aquisição, armazenamento, processamento e análise de imagens infravermelhas denominado ThermaCamTM Researcher Pro 2.9.

As imagens infravermelhas serão adquiridas em ambiente climatizado e com controle digital de temperatura e umidade. Tal câmara possui cinco sensores de temperatura e umidade dispostos da seguinte maneira: um no teto (central), e outros quatro, sendo instalado um em cada parede da sala. Os sensores são conectados diretamente a um computador que mantém temperatura e umidade controladas automaticamente por intermédio do software SITRAD®.

Para testar a normalidade da distribuição dos dados, sua tabulação e análise será feito pelo software SPSS (IBM Statistical Package for the Social Sciences - SPSS Statistics 24) as variáveis estudadas serão testadas com a aplicação do Teste de Kolmogorov-Smirnov. E para o coeficiente de correlação linear de Pearson, e o teste t pareado, para comparação entre as médias das variáveis estudadas. 


\section{RESULTADOS ESPERADOS}

Visto que o Karate é um esporte que em combate oficiais, os atletas tem o contato físico, o objetivo e os resultados esperados é que durante o processo de treinamento haja um desgaste muscular onde seja identificado pelas imagens Termográficas, verificando a veracidade se o processo de inflamação muscular no processo de treinamento e depois analisado logo após uma competição, para assim seja feitas as intervenções para não levar a um futuro dano Muscular. Pesquisas mostram que a principal vantagem da termografia é a sua segurança, contudo, sua desvantagem resulta das suas limitações físicas. A técnica bidimensional e não radiante fornece informações sobre estruturas superficiais. Deve-se lembrar que o uso da termografia na medicina do esporte e ou na análise de danos musculares, não pode substituir o exame clínico, porém complementar a avaliação e dar suporte às decisões.

\section{REFERÊNCIAS BIBLIOGRÁFICAS}

BANDEIRA F, Neves EB, Moura MAMd, Nohama P. A termografia no apoio ao diagnóstico de lesão muscular no esporte. Revista Brasileira de Medicina do Esporte. 2014;20(1):42-7.

BANDEIRA, F., et al. (2012). Pode a termografia auxiliar no diagnóstico de lesões musculares em atletas de futebol? Rev Bras Med Esporte, vol.18, no.4. São Paulo jul./ago.

BANDEIRA, Fábio et al. Can thermography aid in the diagnosis of muscle injuries in soccer athletes?. Revista Brasileira de Medicina Do Esporte, v. 18, n. 4, p. 246-251, 2012.

BANDEIRA, Fábio Henrique. A termografia no apoio ao diagnóstico de lesão muscular no esporte. 2013. Dissertação de Mestrado. Universidade Tecnológica Federal do Paraná.

BARREIRA, C. R. A. e MASSIMI, M. A Moralidade e a Atitude Mental no Karatê Do no Pensamento de Gichin Funakoshi. Memorandum, 2, 39- 54. 2002. Disponível em: http://www.fafich.ufmg.br/ memorandum/artigos02/barreira01.htm. Acesso em 27 de outubro de 2016.

BRANCACCIO, P.; LIMONGELLI, F. M.; MAFFULLI, N. Monitoring of serum enzymes in sport. British journal of sports medicine, v. 40, n. 2, p. 96-97, 2006.

BRANCACCIO, Paola; MAFFULLI, Nicola; LIMONGELLI, Francesco Mario. Creatine kinase monitoring in sport medicine. British medical bulletin, v. 81, n. 1, p. 209, 2007.

BRIOSCHI, Marcos Leal; ABRAMAVICUS, Samuel; CORRÊA, Claudio Fernandes. Valor da imagem infravermelha na avaliação da dor. Rev Dor, v. 6, n. 1, p. 514-24, 2005.

CARMONA PMG. Influência de la información termográfica infrarroja en el protocolo de prevención de lesiones de un equipo de fútbol profesional español.(Influence of infrared thermographic information in the injury prevention protocol of a professional spanish footbal team). 2012. Tesis doctorales. 2012.

CHUDECKA M, Lubkowska A. The use of thermal imaging to evaluate body temperature changes of athletes during training and a study on the impact of physiological and morphological factors on skin temperature. Human Movement. 2012;13(1):33-9.

CONFEDERAÇÃO DE KARATÊ BRASILEIRA - Karate Do - 2013 - Disponível em: http://www.karatedobrasil.com/histria. Acesso em: 30 de maio de 2017. 
CORTE, Ana Carolina Ramos e; HERNANDEZ, Arnaldo José. TERMOGRAFIA MÉDICA INFRAVERMELHA APLICADA À MEDICINA DO ESPORTE. Rev Bras Med Esporte, São Paulo, v. 22, n. 4, p. 315-319, Aug. 2016 . Available from $<$ http://www.scielo.br/scielo.php?script=sci_arttext\&pid=S1517-

86922016000400315\&lng=en\&nrm=iso>. access on $26 \quad$ Feb. 2019. http://dx.doi.org/10.1590/1517-869220162204160783.

CRITCHLEY, G. R.; MANNION, Steve; MEREDITH, C. Injury rates in Shotokan karate. British journal of sports medicine, v. 33, n. 3, p. 174-177, 1999.

CRUZAT, Vinicius Fernandes et al. Current aspects about oxidative stress, physical exercise and supplementation. Revista Brasileira de Medicina do Esporte, v. 13, n. 5, p. 336$342,2007$.

CHAUDHRY, Saira et al. The use of medical infrared thermography in the detection of tendinopathy: a systematic review. Physical Therapy Reviews, v. 21, n. 2, p. 75-82, 2016.

Hildebrandt, Carolin et al. "An overview of recent application of medical infrared thermography in sports medicine in Austria" Sensors (Basel, Switzerland) vol. 10,5 (2010): 4700-15.

DE MEIRA, LEANDERSON FRANCO ET AL. TERMOGRAFIA NA ÁREA BIOMÉDICA. PAN AMERICAN JOURNAL OF MEDICAL THERMOLOGY, [S.L.], V. 1, N. 1, P. 31-41, AGO. 2014. ISSN 2358-4696. DISPONÍVEL EM: <HTTP://ABRATERM.COM.BR/REVISTA/INDEX.PHP/PAJTM/ARTICLE/VIEW/12>. ACESSO EM: 26 FEV. 2019. DOI: HTTP://DX.DOI.ORG/10.18073/23584696/PAJMT.V1N1P31-41.

DE OLIVEIRA, Daniella Araújo; VIEIRA, A.; VALENÇA, Marcelo Moraes. Trauma cranio-encefalico e outras lesoes em atletas do karate de alto nivel. Neurobiologia, v. 74, n. 1, p. 107-14, 2011.

DE SOUZA, Cláudio Teodoro et al. Avaliação sérica de danos musculares e oxidativos em atletas após partida de futsal. Rev Bras Cineantropom Desempenho Hum, v. 12, n. 4, p. 269$274,2010$.

FELISBERTO, Ivo Miguel Vieira. Efeito da intensidade do exercício de treino de força na frequência cardíaca e temperatura da pele durante e após o exercício. 2016. Dissertação de Mestrado.

Ferreira, JJA, Mendonça, LCS, Nunes, LAO et al. Ann Biomed Eng (2008) 36: 1420. https://doi.org/10.1007/s10439-008-9512-1

https://wkf.net/karateprotections/ Acesso em: 30 de maio de 2017

JUNIOR, Nelson Kautzner Marques. Local das lesões no karateca masculino do estilo shotokan. EFDeportes.com, Revista Digital. Buenos Aires, Año 17, Nº 173, Octubre de 2012. http://www.efdeportes.com/

KITCHEN, S. et al. Reparo dos tecidos. Kitchen, S, Bazin S. Eletroterapia de Clayton. São Paulo: Manole, 1998.

LAGE, Victor; GONÇALVES JUNIOR, Luiz. Karatê-do como própria vida. Motriz. Revista de Educação Física. UNESP, v. 13, n. 1, p. 33-42, 2007.

LAUTERT, RICARDO WALTER et al. As artes marciais no caminho do guerreiro: novas possibilidades para o karatê-do. Práticas Corporais, p. 135, 2005. 
MILANEZ, Vinicius Flavio et al. Resposta da frequência cardíaca durante sessão de treinamento de karatê. Rev. Bras. Med. Esporte, São Paulo, v. 18, n. 1, p. 42-45, fev. 2012.

NEVES, Eduardo Borba et al. The influence of subcutaneous fat in the skin temperature variation rate during exercise. Res. Bioma. Eng., Rio de Janeiro, v. 31, n. 4, p. 307-312, Dec. 2015 .

NEVES, Eduardo Borba et al. Using skin temperature and muscle thickness to assess muscle response to strength training. Rev. Bras. Med. Esporte, São Paulo, v. 21, n. 5, p. 350354, Oct. 2015.

NEVES, Eduardo Borba, Reis VM. Fundamentos da termografia para o acompanhamento do treinamento desportivo. Rev Uniandrade. 2014;15(2):79-86

NEVES, Eduardo Borba; REIS, Victor Machado. Fundamentos da termografia para o acompanhamento do treinamento desportivo. Revista Uniandrade, v. 15, n. 2, p. 79-86, 2014.

ROSSI, Luciana; TIRAPEGUI, Julio. Avaliação antropométrica de atletas de Karatê. Revista Brasileira de Ciência e Movimento, v. 15, n. 3, p. 39-46, 2008.

SANCHES, Ionildo José et al. Fusão 3D de imagens de MRI/CT e termografia. Rev. bras. eng. biomed, v. 29, n. 3, p. 298-308, 2013.

SANTINA, Lucas Della; PIMENTA, Thiago Farias da Fonseca. RELAÇÃO ENTRE OS NÍVEIS DE FLEXIBILIDADE COM O DESEMPENHO DOS ATLETAS NAS COMPETIÇÕES DE KARATÊ NA MODALIDADE KATA. Anais do EVINCI-UniBrasil, v. 2, n. 1, p. 152-152, 2016.

SANTOS, Paulo José Moraes de Paula. Análise biomecânica do chute frontal de karatê: implicações em lesões nos membros inferiores. 2015. 75 f. Dissertação (Mestrado) Universidade Estadual Paulista. Faculdade de Engenharia de Guaratinguetá, 2015. Disponível em: 〈http://hdl.handle.net/11449/123240>.

SOUZA, José Mario Couto de et al . Lesões no Karate Shotokan e no Jiu-Jitsu: trauma direto versus indireto. Rev Bras Med Esporte, São Paulo, v. 17, n. 2, p. 107-110, Apr. 2011 - Available from <http://www.scielo.br/scielo.php?script=sci_arttext\&pid=S1517$86922011000200007 \& \operatorname{lng}=\mathrm{en} \& \mathrm{nrm}=\mathrm{iso}>$. access on 05 June 2017. http://dx.doi.org/10.1590/S1517-86922011000200007.

TAN, Jen-Hong et al. Infrared thermography on ocular surface temperature: a review. Infrared physics \& technology, v. 52, n. 4, p. 97-108, 2009.

Verkhoshanski YV. Treinamento Desportivo: Teoria e Metodologia. Porto Alegre: Artmed, 2001

VERKHOSHANSKI, Yuri V. et al. Treinamento desportivo: teoria e metodologia. ArtMed, 2000.

WEINECK, Erlangen J. Futebol total: o treinamento físico no futebol. Phorte, 2000.

WILMORE, Jack H. Fisiologia do esporte e do exercício. Manole, 2001.

ZETARUK, et al. Injuries in martial arts: a comparison of five styles. British Journal of Sports Medicine. London, v. 39, n. 2, p. 29 - 33, 2005.

Ziaee, Vahid et al. "Sport Injuries of Karate During Training: An Epidemiologic Study in Iran." Asian Journal of Sports Medicine 6.2 (2015): e26832. PMC. Web. 5 June 2017. 